\title{
Comparison of the Effects of Epicardial and Endocardial Cardiac Resynchronization Therapy on Transmural Dispersion of Repolarization
}

\author{
EMIN EVREN ÖZCAN, M.D., * SZABOLCS SZILAGYI, M.D., * ZOLTAN SALLO, M.D., * \\ LEVENTE MOLNAR, M.D., * ENDRE ZIMA, M.D., Ph.D., * GABOR SZEPLAKI, M.D., PH.D., * \\ ISTVAN OSZTHEIMER, M.D., * ALI ÖZTÜRK, M.D.,† BÉLA MERKELY, M.D., PH.D., D.Sc., * \\ and LASZLO GELLER, M.D., PH.D.* \\ From the *Heart Center, Semmelweis University, Budapest, Hungary; and tDepartment of Cardiology, Sifa \\ University, Izmir, Turkey
}

Background: Despite significant improvements in cardiac output and functional capacity with cardiac resynchronization therapy (CRT), incidence of sudden cardiac death still remains high. Reversal of physiological myocardial activation sequence during epicardial pacing increases the transmural dispersion of repolarization (TDR). The aim of this study was to compare the effects of endocardial and epicardial biventricular pacing on repolarization parameters in the same patient group.

Methods: Seven patients who had transseptal endocardial left ventricle (LV) lead placement, in whom epicardial CRT had failed due to coronary sinus (CS) lead dislodgement after successful implantation, were admitted to the study. LV endocardial leads were implanted through the interatrial septum in a lateral position. Electrocardiograms (ECGs) were scanned before and after successful epicardial and endocardial biventricular pacing and analyzed using digital calipers. ECG markers of TDR (TpTe and TpTe/QT ratio) were measured and compared.

Results: Baseline QRS durations (161.7 $\pm 15.9 \mathrm{~ms}$ vs $162.2 \pm 17.8 \mathrm{~ms}, P=0.95)$, TpTe values (107.1 \pm $20.5 \mathrm{~ms}$ vs $108.5 \pm 17.6 \mathrm{~ms}, P=0.89)$, and $\mathrm{TpTe} / \mathrm{QT}$ ratios $(0.24 \pm 0.05$ vs $0.24 \pm 0.03, P=0.88)$ were similar before epicardial and endocardial CRT. QRS interval reduction was similar (-28.3 $\pm 11.6 \mathrm{~ms}$ vs $-29.1 \pm 11.4 \mathrm{~ms}, P=0.89$ ) in both groups. Compared to transseptal endocardial CRT, epicardial CRT was associated with a significant increase in TpTe (17.1 $\pm 19.5 \mathrm{~ms} v \mathrm{vs}-12.6 \pm 18.9 \mathrm{~ms}, \mathrm{P}=0.01)$ and TpTe/QT ratio $(0.03 \pm 0.04$ vs $-0.02 \pm 0.03, P=0.04)$.

Conclusion: Transseptal LV endocardial pacing is associated with significant reduction in TDR characteristics compared to epicardial pacing in CRT. Further studies are warranted to determine whether these effects may contribute to reduction of arrhythmias in patients with CRT. (PACE 2015; 0:1-7)

cardiac resynchronization therapy, transmural dispersion of repolarization, transseptal endocardial biventricular pacing, heart failure, ventricular arrhythmias

\section{Introduction}

Despite all the advances in pharmacological treatment, heart failure (HF) remains a leading cause of morbidity and mortality. ${ }^{1-3}$ For this reason, cardiac resynchronization therapy (CRT) is an important therapeutic option in the management of patients with symptomatic systolic $\mathrm{HF}^{4,5}$ Biventricular pacing significantly improves

\footnotetext{
This work was supported by the János Bolyai Research Scholarship of the Hungarian Academy of Sciences (GSZ, LG) and the Hungarian Scientific Research Found (OTKA 105555).

Address for reprints: Laszlo Geller, M.D., Ph.D., Heart Center, Semmelweis University, Gaál József street 9, 1122, Budapest, Hungary. Fax: 36-1-4586842; e-mail: laszlo.geller@gmail.com

Received February 21, 2015; revised April 22, 2015; accepted May 26, 2015.

doi: 10.1111/pace.12678
}

cardiac output, quality of life, and functional capacity in patients with congestive HF. However, the incidence of sudden cardiac death still remains high. ${ }^{6}$ Recent studies have shown that reversal of the normal myocardial activation sequence during epicardial pacing, as it occurs during conventional CRT, increases the transmural dispersion of repolarization (TDR). ${ }^{7,8}$ Biventricular pacing or left ventricular (LV) epicardial pacing may increase the QT interval and TDR, which have the potential to increase the risk of ventricular arrhythmias. ${ }^{8-10}$ Increased TDR as measured by Tpeak-Tend (TpTe) and TpTe/QT is found to be associated with a higher incidence of ventricular arrhythmias in cardiac resynchronization therapy with defibrillator (CRT-D) patients. ${ }^{11}$ Although large multicenter randomized studies have not reported any increased incidence of ventricular tachycardia (VT) or ventricular fibrillation (VF)

(C) 2015 Wiley Periodicals, Inc. 
episodes, it is important to determine CRT patients who are prone to have ventricular arrhythmias. ${ }^{4,6}$

CRT with transseptal endocardial LV pacing is an alternative in patients where the conventional approach has failed. ${ }^{12,13}$ Endocardial pacing also leads to more physiological activation. Experimental observations suggest that potential arrhythmias can be avoided by stimulation of the LV endocardium. ${ }^{9}$ A recently published small case control study suggests that permanent biventricular pacing with LV endocardial lead placement through the interatrial septum is associated with significantly lower TpTe and QT dispersion values compared to control group with a coronary sinus (CS) LV lead. ${ }^{14}$ This small observational study with a case control design provides crucial information. However, finding a perfect match between small patient groups is not always possible and differences in baseline characteristics between groups may have an impact on results. The aim of this study was to compare the effects of endocardial and epicardial biventricular pacing on repolarization characteristics in the same patient group.

\section{Methods}

\section{Patient Population}

All patients suitable for transseptal endocardial LV lead placement, in whom epicardial CRT had failed due to lead dislodgement after successful CS implantation, were admitted to the study. All patients included were required to meet European Society of Cardiology Class I criteria for CRT implantation. ${ }^{4}$ After successful transseptal CRT implantation, all patients' hospital recordings were evaluated. Patients who had previous successful conventional CRT implantation were selected. Electrocardiograms (ECGs) were recorded before and after successful conventional CRT, as were location of CS leads and pacing threshold values.

\section{Endocardial LV Lead Implantation}

Procedures started with transseptal puncture from the femoral vein, under guidance of intracardiac echocardiography. The puncture site was dilated with an 8-mm angioplasty balloon, and the guidewire was left across the septum as a marker. Subsequently, a guidewire was passed through the same hole inserted from the subclavian vein through a guiding catheter produced for CS lead implantation. Once the guiding catheter was advanced to the left atrium, its tip was directed to the mitral valve and an active fixation lead was implanted in the lateral area of the LV. All patients were maintained on anticoagulation therapy with either warfarin or coumarin.

\section{Electrocardiographic Measurements}

ECGs $(25 \mathrm{~mm} / \mathrm{s}, 10 \mathrm{~mm} / \mathrm{mV})$ were recorded on admission (preendocardial CRT ECG [pre-endoCRT ECG]) and before discharge (postendocardial CRT ECG [post-endo-CRT ECG]) after successful LV endocardial lead implantation. The ECGs of the same patients before and after successful CRT with CS epicardial pacing were found from hospital records. These ECGs were defined as preepicardial CRT (pre-epi-CRT) and postepicardial CRT (postepi-CRT) ECGs. All ECGs were digitally scanned and measurements were made with digital calipers at $400 \%$ magnification. Analysis was performed by a blinded physician. Lead V5 was selected for analysis. If the $\mathrm{T}$ wave in V5 was not eligible, lead II was used. The QT interval was defined as the time interval between the initial deflection of the QRS and the point at which a tangent drawn on the steepest downslope of the T wave crossed the isoelectric line. ${ }^{15}$ Intervals were corrected for heart rate using Bazett's formula. ${ }^{16}$ The TpTe interval was obtained from the difference between the QT interval and the QT peak interval. The QT peak interval was measured from the beginning of the QRS until the peak of a positive $\mathrm{T}$ wave or the nadir of a negative $\mathrm{T}$ wave. ${ }^{17}$ The TpTe/QT ratio was also calculated. Patients were on the same medications during measurements and none of them had anesthesia during procedures.

\section{Statistics}

Mean and standard deviations (SD) were used for descriptive statistics. Categorical data were summarized as frequencies and percentages. Comparisons of repolarization parameters between pacing modes (baseline, epi-CRT, endoCRT) were performed with paired two-tailed Student $t$-tests. In all analyses, $\mathrm{P}<0.05$ was considered statistically significant.

\section{Results}

Between September 2007 and March 2014, 48 patients in our center had transseptal endocardial LV lead placement. Thirteen of them had a CS lead dislodgement history after successful conventional CRT implantation and epicardial pacing. Five patients who did not have analyzable CRT ECGs and one patient who had CRT upgrade from RV pacing were excluded. Therefore, seven patients were included. No patient was excluded due to uncontrolled heart rate (biventricular pacing rate $<90 \%$ ), procedure-related complications, or death. Baseline characteristics are given in Table I. The mean age of the patients was $63 \pm 4$ years and the majority of them were male. Four $(57.1 \%)$ had ischemic dilated cardiomyopathy. The positions of the CS leads 
Table I.

Baseline Characteristics of Study Patients

\begin{tabular}{lc}
\hline Age (years) & $63 \pm 3.7$ \\
Male & $6(85.7)$ \\
LVEF (\%) & $30 \pm 3.0$ \\
Etiology & \\
$\quad$ Ischemic & $4(57.1)$ \\
$\quad$ Nonischemic & $3(42.9)$ \\
NYHA functional class & \\
II & $1(14.3)$ \\
III & $6(85.7)$ \\
Device & \\
CRT-D & $4(57.1)$ \\
CRT-P & $3(42.9)$ \\
AF & $2(28.6)$ \\
DM & $1(14.3)$ \\
HT & $5(71.4)$ \\
Hg (g/dL) & $14.4 \pm 1.4$ \\
Cr ( $\mu$ mol/L) & $89.2 \pm 14.6$ \\
Drugs & \\
ACE-I/ARB & $7(100)$ \\
$\beta$-Blocker & $5(71.4)$ \\
Amiodarone & $1(14.3)$ \\
Other QT prolonging drug & 0 \\
QRS morphology (LBBB) & $7(100)$ \\
\hline Valus are mean & \\
\hline
\end{tabular}

Values are mean \pm SD or $\mathrm{n}(\%)$.

ACE-I = angiotensin-converting enzyme inhibitor; $\mathrm{AF}=$ atrial fibrillation; $\mathrm{ARB}=$ angiotensin $\mathrm{II}$ receptor blocker; CRT-D = cardiac resynchronization therapy with defibrillator; CRT-P = cardiac resynchronization therapy pacemaker; $\mathrm{DM}=$ diabetes mellitus; $\mathrm{HT}=$ hypertension; LBBB = left bundle branch block; $\mathrm{LVEF}=$ left ventricle ejection fraction; NYHA = New York Heart Association.

were reported in a lateral location with stable pacing thresholds. Transseptal LV leads were implanted in lateral position in all patients. Mean elapsed time between the initial epicardial CRT and endocardial CRT was $10.5 \pm 8.9$ months. No thromboembolic complications were observed during the follow-up.

Baseline QRS durations $(161.7 \pm 15.9 \mathrm{~ms}$ vs $162.2 \pm 17.8 \mathrm{~ms}, \mathrm{P}=0.95)$, QTc intervals (435.6 \pm $56.9 \mathrm{~ms}$ vs $444.6 \pm 44.5 \mathrm{~ms}, \mathrm{P}=0.73)$, TpTe values $(107.1 \pm 20.5 \mathrm{~ms}$ vs $108.5 \pm 17.6 \mathrm{~ms}, \mathrm{P}=$ $0.89)$, and TpTe/QT ratios (0.24 \pm 0.05 vs $0.24 \pm$ $0.03, \mathrm{P}=0.88$ ) were similar before epi- and endo-CRT (Table II).

In all patients, QRS intervals reduced significantly following both epi- and endo-CRT $(161.71 \pm 16 \mathrm{~ms}$ vs $133.42 \pm 15 \mathrm{~ms}, \mathrm{P}<0.01$; $162.28 \pm 18 \mathrm{~ms}$ vs $133.14 \pm 8 \mathrm{~ms}, \mathrm{P}<0.01$, respectively). Although QRS interval reductions were similar $(-28.3 \pm 11.6 \mathrm{~ms}$ vs $-29.1 \pm 11.4 \mathrm{~ms}$, $\mathrm{P}=0.89$ ), epi-CRT was associated with a

\section{Table II.}

Comparison of Repolarization Parameters before and after Cardiac Resynchronization Therapy with

Endocardial and Epicardial Left Ventricular Pacing

\begin{tabular}{lrcc}
\hline & Epicardial & Endocardial & P Value \\
\hline Baseline QRS & $161.7 \pm 15.9$ & $162.2 \pm 17.8$ & 0.95 \\
Baseline QTc & $435.6 \pm 56.9$ & $444.6 \pm 44.5$ & 0.73 \\
Baseline & $107.1 \pm 20.5$ & $108.5 \pm 17.6$ & 0.89 \\
$\quad$ Tp-Te & & & \\
Baseline & $0.24 \pm 0.05$ & $0.24 \pm 0.03$ & 0.88 \\
$\quad$ Tp-Te/QT & & & \\
$\begin{array}{l}\text { Post CRT } \\
\text { Tp-Te }\end{array}$ & $124.3 \pm 30.8$ & $96.0 \pm 27.1$ & 0.09 \\
Post CRT & $0.27 \pm 0.05$ & $0.22 \pm 0.04$ & 0.08 \\
$\quad$ Tp-Te/QT & & & \\
$\begin{array}{l}\Delta \text { QRS } \\
\Delta \text { QTc }\end{array}$ & $-28.3 \pm 11.6$ & $-29.1 \pm 11.4$ & 0.89 \\
$\Delta$ Tp-Te & $14.4 \pm 37.5$ & $-17.0 \pm 45.2$ & 0.19 \\
$\Delta$ Tp-Te/QT & $0.03 \pm 0.04$ & $-0.02 \pm 0.03$ & 0.04 \\
\end{tabular}

$\mathrm{TpTe}=$ Tpeak-Tend

significant increase in TpTe values $(17.1 \pm 19.5$ $\mathrm{ms}$ vs $-12.6 \pm 18.9 \mathrm{~ms}, \mathrm{P}=0.01)$ and TpTe/QT ratios $(0.03 \pm 0.04$ vs $-0.02 \pm 0.03, \mathrm{P}=0.04)$ compared to endo-CRT (Fig. 1). Differences in QTc interval changes were not significant (14.4 \pm $37.5 \mathrm{~ms}$ vs $-17.0 \pm 45.2 \mathrm{~ms}, \mathrm{P}=0.19$; Table II). RR intervals were similar before and after, epi- and endo-CRT (pre-epi vs pre-endo, $797.14 \pm 55 \mathrm{~ms}$ vs $806.00 \pm 45 \mathrm{~ms}, \mathrm{P}=0.85$, post-epi vs post-endo, $781.12 \pm 73 \mathrm{~ms}$ vs $786.24 \pm 46 \mathrm{~ms}, \mathrm{P}=0.87$ ).

\section{Discussion}

The main findings of this study suggest that permanent LV endocardial CRT is associated with significant reduction in TDR characteristics compared to conventional epicardial CRT. Nonphysiological activation of both ventricles with different transmural activation sequences might be responsible for an increase in TDR during epicardial CRT. Our study is the first to compare the effects of permanent endocardial and epicardial CRT in the same patient group. The effect of stimulating both sides of the same substrate (the lateral wall of the LV) was evaluated.

It has been shown that even in the absence of any difference in final repolarization time, reversing the direction of activation affects the action potential curve and T-wave morphology. ${ }^{8,9}$ Medina-Ravell et al. evaluated pacing site dependent changes in ventricular repolarization, and observed more significant prolongation in TDR with epicardial and biventricular pacing. ${ }^{7}$ They 


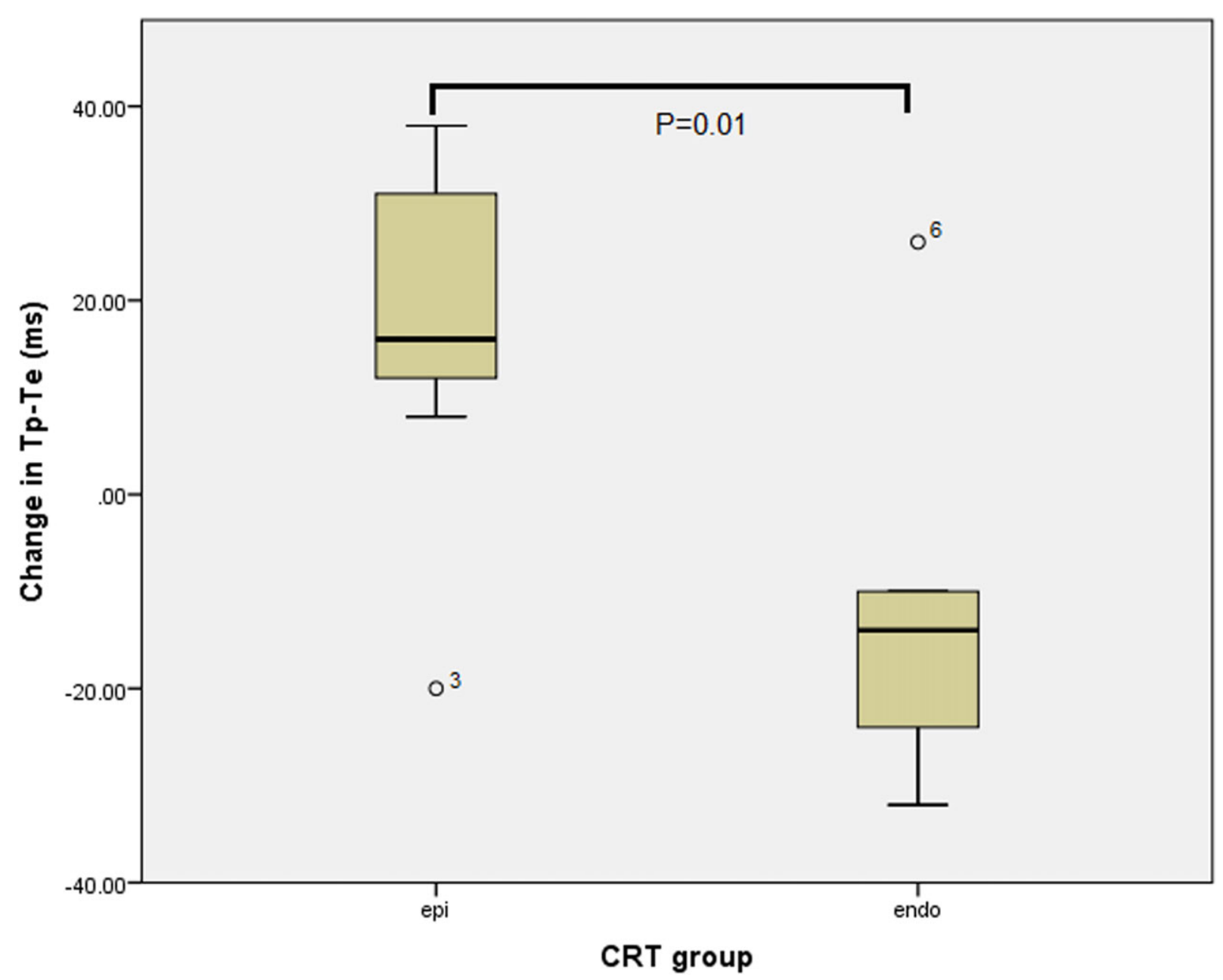

Figure 1. Box plot of change in TpTe following epicardial and endocardial cardiac resynchronization therapy. $C R T=$ cardiac resynchronization therapy; endo = endocardial; epi $=$ epicardial; $T p T e=T p e a k-T e n d$.

reported the development of torsades de pointes in a HF patient with epicardial or biventricular pacing, but not with endocardial pacing only. Monomorphic ventricular tachycardias induced by CRT are also reported. ${ }^{18,19}$

Despite of the few case reports, none of the large multicenter randomized trials have reported an increased risk of ventricular arrhythmias with CRT. ${ }^{20-24}$ Higgins et al. observed less implantable cardioverter defibrillator (ICD) therapy with CRT compared to no pacing. ${ }^{20}$ A metaanalysis of randomized trials of CRT also found no relation between sudden cardiac death and CRT. ${ }^{21}$ Kutyifa et al. analyzed the association between the LV lead location and the risk of ventricular arrhythmias in patients enrolled in the Multicenter Automatic Defibrillator Implantation Trial with Cardiac Resynchronization Therapy (MADIT-CRT) trial. ${ }^{22}$ Posterior and lateral LV lead location was associated with decreased risk of ventricular arrhythmias in comparison with an anterior lead location or ICD-only group.

These results raise concern that CRT might have a differential effect on different substrates and also pacing site might have an impact. ${ }^{23}$ Recently, Scott et al. investigated the effect of permanent endocardial CRT on ventricular repolarization in humans. ${ }^{14}$ They evaluated seven patients with transseptal $\mathrm{LV}$ endocardial leads, 28 matched patients with CS LV leads, and eight patients with surgical LV epicardial leads. Significant postpacing reduction in TpTe and QT dispersion values were observed in the transseptal group compared to the CS group. However, this case control designed study had limitations due to the difficulty of finding a perfect match between the groups. Although our observations on repolarization patterns were consistent with 

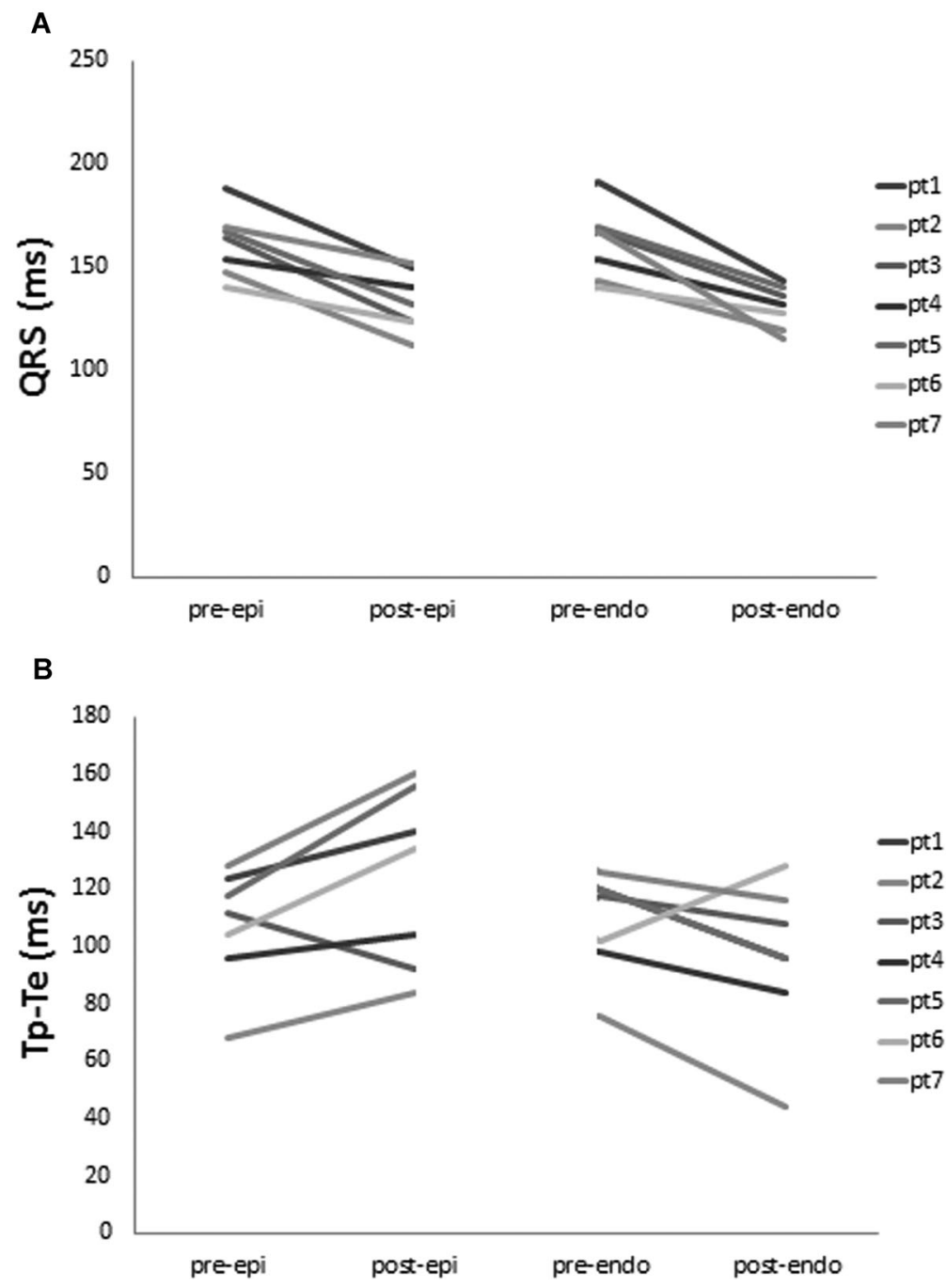

Figure 2. The effects of epicardial and endocardial cardiac resynchronization therapy (CRT) on QRS (A) and $T p T e(B)$ in the same patient group. Please note that epicardial and endocardial CRT produced similar QRS interval reductions. However, epicardial CRT was associated with a significant increase in TpTe values compared to endocardial CRT . post-endo = postendocardial CRT; post-epi = postepicardial CRT; pre-endo = preendocardial CRT; pre-epi $=$ preepicardial CRT; TpTe $=$ Tpeak-Tend .

Scott et al., we observed different depolarization changes. They reported significantly better postCRT reduction in QRS duration in the transseptal group. However, we observed similar QRS reduction with epi- and endo-CRT in the same patient group.

The QRS interval during biventricular pacing is complicated because there are two activation vectors. The underlying heart disease and localizations of myocardial scars can make a contribution to the electrophysiological effects of biventricular pacing. Therefore, differences between the $\mathrm{LV}$ endocardial patients and the control group might be responsible for this finding.
Factors that influence depolarization patterns may also affect repolarization patterns. Nevertheless, our results corroborate animal experiments which evaluated the effects of endocardial and epicardial pacing on transmural wedge preparations. $^{7,25}$ Despite a similar reduction in QRS intervals, we observed significant changes in TpTe and TpTe/QT values (Fig. 2). QTc and TpTe values were increased with epi-CRT and decreased with endo-CRT. Therefore, the main differential effect of endocardial CRT seems to be on repolarization rather than to depolarization.

A TpTe/QT ratio of $\geq 0.25$ is reported as a significant risk factor for appropriate ICD 
therapy in conventional CRT-D patients. ${ }^{11}$ In our patient group, baseline $\mathrm{TpTe} / \mathrm{QT}$ ratios were similar for both epi- and endo-CRT. However, mean TpTe/QT ratios increased to $0.27 \pm 0.05$ with epi-CRT and decreased to $0.22 \pm 0.04$ with endo-CRT. $\triangle \mathrm{TpTe} / \mathrm{QT}$ values were significantly different (Table II).

However, in two patients different responses were observed (Fig. 2). In patient 3, TpTe values were decreased both after epicardial and endocardial CRT. Contrarily, patient 6 demonstrated TpTe increase with both epicardial and endocardial CRT. Patient 3 had nonishemic cardiomyopathy and relatively better ejection fraction (33\%). Patient 6 had ischemic cardiomyopathy with large anterior scar and ejection fraction was $27 \%$. Although LV was stimulated in the same region but opposite sites, the presence of ischemic scars and heterogeneity of the myocardial substrate may lead to changes in conduction vectors and may change the transmural repolarization sequence. Impact of epicardial pacing on heterogenous myocardium is more prominent and electrical heterogeneity is amplified when normal activation of ventricular wall is reversed. ${ }^{8}$ Unfortunately, due to the relatively small number of patients, no subgroup analysis on ischemic and nonischemic patients was performed.

$\mathrm{LV}$ pacing region and pacing rate might have an impact on TDR. Both epicardial and endocardial leads were implanted in the lateral region of the LV and RR intervals were similar during measurements. Another factor that may influence the TDR is the interventricular (VV) pacing delay. In our study, VV timing intervals were optimized to achieve best QRS duration. Therefore, VV timing intervals were not uniform across the patient population. However, this is an accepted CRT optimization algorithm and reflects the daily practice.

\section{References}

1. McMurray JJ, Adamopoulos S, Anker SD, Auricchio A, Böhm M, Dickstein K, Falk V, et al. ESC guidelines for the diagnosis and treatment of acute and chronic heart failure 2012: The Task Force for the Diagnosis and Treatment of Acute and Chronic Heart Failure 2012 of the European Society of Cardiology. Developed in collaboration with the Heart Failure Association (HFA) of the ESC. Eur Heart J 2012; 33:1787-1847.

2. vonScheidt W, Zugck C, Pauschinger M, Hambrecht R, Bruder O, Hartmann A, Rauchhaus $\mathrm{M}$, et al. Characteristics, management modalities and outcome in chronic systolic heart failure patients treated in tertiary care centers: Results from the EVIdence based TreAtment in Heart Failure (EVITA-HF) registry. Clin Res Cardiol 2014; 103:1006-1014.

3. Zugck C, Franke J, Gelbrich G, Frankenstein L, Scheffold T, Pankuweit S, Duengen HD, et al. Implementation of pharmacotherapy guidelines in heart failure: Experience from the German Competence Network Heart Failure. Clin Res Cardiol 2012; 101:263-272

4. Brignole M, Auricchio A, Baron-Esquivias G, Bordachar P, Boriani G, Breithardt OA, Cleland J, et al. 2013 ESC guidelines on cardiac pacing and cardiac resynchronization therapy: The Task Force on Cardiac Pacing and Resynchronization Therapy of the European
Our study focused on TDR response during epi- and endo-CRT, and was neither designed nor powered to evaluate the relevant clinical arrhythmias. Our patient group was too small to demonstrate a significant difference in terms of relevant clinical arrhythmias. It is important to stress that our findings reflecting the effects of endocardial CRT on repolarization do not suggest that epicardial CRT is proarrhythmic.

\section{Limitations}

Although our study was limited by the small sample size, it was sufficient to show significant changes in TDR parameters during endo- and epi-CRT in a uniform pattern. However, further multicenter studies with larger patient groups are warranted to confirm these findings.

We selected only lead V5 for repolarization parameter measurements. Analysis of a single lead might influence the accuracy of ventricular repolarization. However, previous clinical studies that showed the association of increased TpTe interval and TpTe/QT ratio and ventricular arrhythmias during CRT also used same measurement technique, and these parameters are widely accepted. ${ }^{11,26,27}$

In addition, only acute responses to CRT were examined in our study, but long-term electrical and mechanical remodeling could modify the results. ${ }^{10,28,29}$

\section{Conclusion}

Transseptal LV endocardial pacing is associated with significant reduction in TDR characteristics compared to epicardial pacing in CRT. Larger clinical studies are needed to determine whether these effects may contribute to reduction of arrhythmias.
Society of Cardiology (ESC). Developed in collaboration with the European Heart Rhythm Association (EHRA). Europace 2013; 15:1070-1118.

5. Wasmer K, Köbe J, Andresen D, Zahn R, Spitzer SG, Jehle J, Brachmann J, et al. Comparing outcome of patients with coronary artery disease and dilated cardiomyopathy in ICD and CRT recipients: Data from the German DEVICE-registry. Clin Res Cardiol 2013; 102:513-521.

6. Cleland JG, Daubert JC, Erdmann E, Freemantle N, Gras D, Kappenberger L, Tavazzi L, et al. Longer-term effects of cardiac resynchronization therapy on mortality in heart failure [the CArdiac REsynchronization-Heart Failure (CARE-HF) trial extension phase]. Eur Heart J 2006; 27:1928-1932.

7. Medina-Ravell VA, Lankipalli RS, Yan GX, Antzelevitch C, Medina-Malpica NA, Medina-Malpica OA, Droogan C, et al. Effect of epicardial or biventricular pacing to prolong QT interval and increase transmural dispersion of repolarization: Does resynchronization therapy pose a risk for patients predisposed to long QT or torsade de pointes? Circulation 2003; 107:740746.

8. Fish JM, Di Diego JM, Nesterenko V, Antzelevitch C. Epicardial activation of left ventricular wall prolongs QT interval and transmural 


\section{CRT AND TRANSMURAL DISPERSION OF REPOLARIZATION}

dispersion of repolarization: Implications for biventricular pacing. Circulation 2004; 109:2136-2142.

9. Fish JM, Brugada J, Antzelevitch C. Potential proarrhythmic effects of biventricular pacing. J Am Coll Cardiol 2005; 46:2340-2347.

10. Wecke L, vanDeursen CJ, Bergfeldt L, Prinzen FW. Repolarization changes in patients with heart failure receiving cardiac resynchronization therapy-signs of cardiac memory. J Electrocardiol 2011; 44:590-598.

11. Barbhaiya C, Po JR, Hanon S, Schweitzer P. T(peak) - T(end) and T(peak) - T(end) /QT ratio as markers of ventricular arrhythmia risk in cardiac resynchronization therapy patients. Pacing Clin Electrophysiol 2013; 36:103-108.

12. Jais P, Douard H, Shah DC, Barold S, Barat JL, Clementy J. Endocardial biventricular pacing. Pacing Clin Electrophysiol 1998; 21:2128-2131.

13. vanGelder BM, Scheffer MG, Meijer A, Bracke FA. Transseptal endocardial left ventricular pacing: An alternative technique for coronary sinus lead placement in cardiac resynchronization therapy. Heart Rhythm 2007; 4:454-460.

14. Scott PA, Yue AM, Watts E, Zeb M, Roberts PR, Morgan JM. Transseptal left ventricular endocardial pacing reduces dispersion of ventricular repolarization. Pacing Clin Electrophysiol 2011; 34:1258-1266.

15. Yamaguchi M, Shimizu M, Ino H, Terai H, Uchiyama K, Oe K, Mabuchi T, et al. Twave peak-to-end interval and QT dispersion in acquired long QT syndrome: A new index for arrhythmogenicity. Clin Sci (Lond) 2003; 105:671-676.

16. Bazett HC. An analysis of the time-relations of electrocardiograms. Heart J 1920; 7:353-370.

17. Letsas K, Weber R, Astheimer K, Kalusche D, Arentz T. TpeakTend interval and Tpeak-Tend/QT ratio as markers of ventricular tachycardia inducibility in subjects with Brugada ECG phenotype. Europace 2010; 12:271-274.

18. Kantharia BK, Patel JA, Nagra BS, Ledley GS. Electrical storm of monomorphic ventricular tachycardia after a cardiacresynchronization-therapy-defibrillator upgrade. Europace 2006; 8:625-628.

19. Shukla G, Chaudhry GM, Orlov M, Hoffmeister P, Haffajee C. Potential proarrhythmic effect of biventricular pacing: Fact or myth? Heart Rhythm 2005; 2:951-956.
20. Higgins SL, Yong P, Sheck D, McDaniel M, Bollinger F, Vadecha M, Desai S, et al. Biventricular pacing diminishes the need for implantable cardioverter defibrillator therapy. Ventak CHF Investigators. J Am Coll Cardiol 2000; 36:824-827.

21. Rivero-Ayerza M, Theuns DA, Garcia-Garcia HM, Boersma E, Simoons M, Jordaens LJ. Effects of cardiac resynchronization therapy on overall mortality and mode of death: A meta-analysis of randomized controlled trials. Eur Heart J 2006; 27:26822688.

22. Kutyifa V, Zareba W, McNitt S, Singh J, Hall WJ, Polonsky S, Goldenberg I, et al. Left ventricular lead location and the risk of ventricular arrhythmias in the MADIT-CRT trial. Eur Heart J 2013; 34:184-190.

23. Leyva F, Foley PW. Is cardiac resynchronisation therapy proarrhythmic? Indian Pacing Electrophysiol J 2008; 8:268280.

24. Birnie DH, Ha A, Higginson L, Sidhu K, Green M, Philippon F, Thibault B, et al. Impact of QRS morphology and duration on outcomes after cardiac resynchronization therapy: Results from the Resynchronization-Defibrillation for Ambulatory Heart Failure Trial (RAFT). Circ Heart Fail 2013; 6:1190-1198.

25. Osadchii OE. Electrophysiological determinants of arrhythmic susceptibility upon endocardial and epicardial pacing in guineapig heart. Acta Physiol (Oxf) 2012; 205:494-506.

26. Lellouche N, DeDiego C, Akopyan G, Boyle NG, Mahajan A, Cesario DA, Wiener I, et al. Changes and predictive value of dispersion of repolarization parameters for appropriate therapy in patients with biventricular implantable cardioverter-defibrillators. Heart Rhythm 2007; 4:1274-1283.

27. Gupta P, Patel C, Patel H, Narayanaswamy S, Malhotra B, Green JT, Yan GX. T(p-e)/QT ratio as an index of arrhythmogenesis. J Electrocardiol 2008; 41:567-574.

28. Braunschweig F, Pfizenmayer H, Rubulis A, Schoels W, Linde C, Bergfeldt L. Transient repolarization instability following the initiation of cardiac resynchronization therapy. Europace 2011; 13:1327-1334.

29. Itoh M, Yoshida A, Fukuzawa K, Kiuchi K, Imamura K, Fujiwara R, Suzuki A, et al. Time-dependent effect of cardiac resynchronization therapy on ventricular repolarization and ventricular arrhythmias. Europace 2013; 15:1798-1804. 